ARTICLE

https://doi.org/10.1038/s41467-019-11087-y

\title{
Dynamic shortening of disorder potentials in anharmonic halide perovskites
}

\author{
Christian Gehrmann ${ }^{1,2}$ \& David A. Egger ${ }^{1,2}$
}

Halide perovskites are semiconductors that exhibit sharp optical absorption edges and small Urbach energies allowing for efficient collection of sunlight in thin-film photovoltaic devices. However, halide perovskites also exhibit large nuclear anharmonic effects and disorder, which is unusual for efficient optoelectronic materials and difficult to rationalize in view of the small Urbach energies that indicate a low amount of disorder. To address this important issue, the disorder potential induced for electronic states by the nuclear dynamics in various paradigmatic halide perovskites is studied with molecular dynamics and density functional theory. We find that the disorder potential is dynamically shortened due to the nuclear motions in the perovskite, such that it is short-range correlated, which is shown to lead to favorable distributions of band edge energies. This dynamic mechanism allows for sharp optical absorption edges and small Urbach energies, which are highly desired properties of any solar absorber material.

\footnotetext{
${ }^{1}$ Institute of Theoretical Physics, University of Regensburg, 93040 Regensburg, Germany. ${ }^{2}$ Department of Physics, Technical University of Munich, 85748 Garching, Germany. Correspondence and requests for materials should be addressed to D.A.E. (email: david.egger@tum.de)
} 
alide perovskites (HaPs) have emerged as semiconducting materials that are solution-processable and show an outstanding potential for device applications, notably for photovoltaics, where power-conversion efficiencies already approach those of silicon-based cells ${ }^{1-10}$. HaPs exhibit several physical properties that are key to their remarkable potential as solar materials, and in particular show a steep optical absorption rise that is required for an efficient capture of sunlight in a thinfilm device ${ }^{11}$. Specifically, the Urbach energy, which quantifies the steepness of optical absorption rise, was found to be very small for HaPs at room temperature, on the order of $10 \mathrm{meV}^{12}$, which is close to highly efficient inorganic solar materials such as bulk $\mathrm{Si}$ and GaAs. A small Urbach energy implies that the material is ordered, since disorder would lead to tail states in the electronic structure and to a broadening of the optical absorption profile. Interestingly, however, ample experimental and theoretical evidence point to highly anharmonic nuclear motion and disorder being active in HaPs at room temperature, which also involves the ions in the crystal contributing to the frontier electronic band structure ${ }^{13-25}$. Such anharmonic effects are uncommon for efficient optoelectronic materials ${ }^{26}$ and difficult to rationalize in view of the low Urbach energy. In particular, they can be suspected to result in a disordered potential for the electrons and holes in the crystal and, hence, in a high density of tail states and a broad optical absorption. Therefore, one must wonder how steep optical absorption edges, small Urbach energies and, hence, an efficient collection of sunlight can even be possible in thin-films of $\mathrm{HaP}$ crystals at room temperature.

Here, we use first-principles based molecular dynamics (MD) calculations to study and quantify the spatial correlations in the disorder potential for electrons and holes that is induced by thermal vibrations. We find that the massive nuclear motions in $\mathrm{HaPs}$ lead to a dynamic shortening of the disorder potential for electrons and holes, such that is confined to one atomic bond and becomes similar to the length scales that were reported for classical inorganic semiconductors. Since the correlation length of the disorder potential is short-ranged, thermal nuclear motion disturbs the electronic states in only small volumes of the crystal. Therefore, this is expected to lead to a low density of disorderinduced tail states, which we demonstrate from first-principles calculations. This dynamic mechanism allows the here studied $\mathrm{CsPbBr}, \mathrm{CsPbI}_{3}$, and $\mathrm{MAPbI}_{3}$ to exhibit narrow band-gap distributions, sharp optical absorption edges and small Urbach energies at elevated temperatures, which are highly desired properties of any solar absorber material used in thin-film devices.

\section{Results}

Spatial correlations in the disorder potential of $\mathrm{CsPbBr}_{3}$. Our theoretical approach employs MD calculations based on density functional theory (DFT), which treat the nuclear anharmonicity to all orders in the Taylor expansion of the crystal potential and thereby allow for monitoring and quantifying its consequences for physical observables in a straightforward manner. Specifically, long MD trajectories at various temperatures were computed in order to characterize the consequences of the thermally-induced nuclear disorder potential for electronic states in the material, as illustrated in Fig. 1a. To this end, we firstly calculated the average electrostatic potential energy as

$$
\bar{V}(x, y, z)=\frac{1}{N} \sum_{i=1}^{N} V_{i}(x, y, z)
$$

where $V_{i}(x, y, z)$ is the electrostatic energy of the electrons for configuration $i$ along the MD trajectory, and $N$ is the total number of configurations we considered. Note that we chose
$N=30$ configurations, which were separated by 5 ps. We consider $\bar{V}$ to represent the average crystal potential for the electronic states. From this, we calculated the instantaneous disorder potential as the deviation from $\bar{V}$ :

$$
\Delta V_{i}(x, y, z)=V_{i}(x, y, z)-\bar{V}(x, y, z)
$$

Finally, we computed the autocorrelation function of $\Delta V_{i}$ along the $y$ coordinate as

$$
C_{i}(x, \Delta y, z)=\frac{<\Delta V_{i}(x, y+\Delta y, z) \Delta V_{i}(x, y, z)>}{<\Delta V_{i}(x, y, z) \Delta V_{i}(x, y, z)>}
$$

and owing to the cubic symmetry of the crystal, calculated the average of it over all $x$ and $z$ values, which we denote as $C(\Delta y)$. Furthermore, the DFT-MD simulations allow for characterizing phonon-quasiparticle properties by analyzing the velocityautocorrelation function (VACF) in the basis of the harmonic spectrum (see Methods section for details). As a paradigmatic case, we choose to focus first on the all-inorganic $\mathrm{HaP} \mathrm{CsPbr}_{3}$ in its cubic phase (Fig. 1a), and subsequently report results on $\mathrm{CsPbI}_{3}$ and $\mathrm{MAPbI}_{3}$ in order to test the influence of ionic composition.

Figure $1 \mathrm{~b}$ shows an exemplary charge-density response during the $\mathrm{MD}$ in $\mathrm{CsPbBr}_{3}$, which was obtained as the difference of the density corresponding to a randomly-chosen nuclear configuration along the MD run at $425 \mathrm{~K}$ and the mean density along the MD trajectory. The calculated charge-density difference is such that it follows the nuclear displacements, and since all phonon modes are excited at the considered temperature (see section "vibrational characterization of $\mathrm{CsPbBr}_{3}$ "), it is essentially distributed throughout the entire simulation volume. What is perhaps more surprising is the fact that this charge-density difference appears highly disordered, i.e., it does not show any obvious symmetry properties or directional features.

Such a charge density response results in an instantaneous disorder potential for the electronic states in $\mathrm{CsPbBr}_{3}$ via Eq. (2) that is spatially correlated by means of $C(\Delta y)$ given in Eq. (3), which we calculated self-consistently along the MD trajectory. $C(\Delta y)$ is shown in Fig. 1c for multiple instantaneous nuclear configurations along the MD trajectory, together with the average of these snapshots, for the three temperatures considered in the MD simulations. Remarkably, it is found that the correlation in the disorder potential is confined to very short ranges, since it vanishes on length scales of atomic bonds in $\mathrm{Cs} \mathrm{PbBr}_{3}$. Furthermore, we find that at the three considered temperatures, the disorder potential shows a similar overall behavior. With this, we establish that the spatial correlation in the disorder potential $\mathrm{CsPbBr}$ is short-ranged, such that it appears to be similar to the one present in classical inorganic semiconductors, which is correlated also only over atomic distances ${ }^{27}$.

Vibrational characterization of $\mathrm{CsPbBr}_{3}$. The short-ranged spatial correlations for the electronic states in $\mathrm{CsPbBr}_{3}$ are at first sight peculiar because the nuclear dynamics driving the disorder in HaPs were reported to be very different compared to the situation in classical inorganic semiconductors ${ }^{13-25}$. We characterize the key vibrational features of $\mathrm{CsPbBr}_{3}$ in the following by first discussing results from the harmonic approximation. This description is valid for materials in which small nuclear displacements occur at finite temperature, as is the case in many of the classical inorganic semiconductors at room temperature. The harmonic phonon spectrum of $\mathrm{CsPbBr}_{3}$ (see Fig. 2a) is found to contain low-energy features, especially due to $\mathrm{Cs}$ and $\mathrm{Br}$ motion, as was well as an intrinsic dynamic instability, which have been analyzed in previous theoretical work $^{28,29}$. Next, we consider 
a

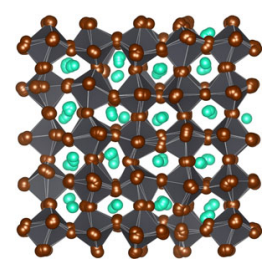

b

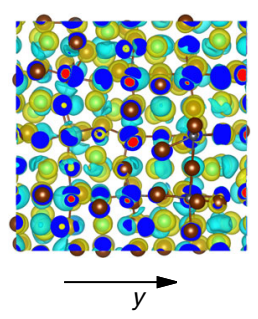

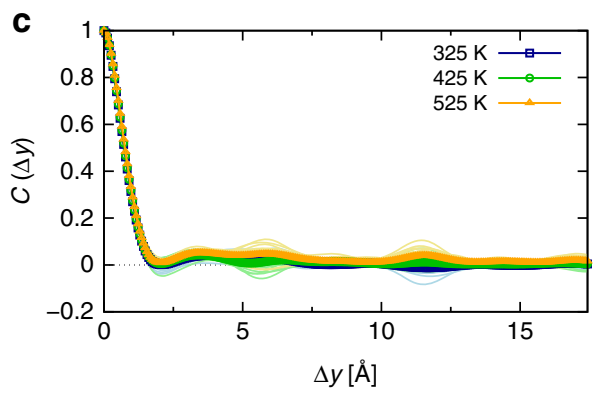

Fig. 1 Characterization of the disorder potential for electronic states in $\mathrm{CsPbBr}_{3}$. a Visualization of fully anharmonic dynamics in $\mathrm{CsPbBr}$, which include phonon-phonon interactions in the finite-temperature description of the dynamic distortions present in the crystal. Cs atoms are shown in cyan, $\mathrm{Pb}$ atoms in gray, and $\mathrm{Br}$ atoms in brown color; the latter two species form gray-colored octahedra. $\mathbf{b}$ Iso-surface representation of the charge-density difference that is induced in the crystal at an instantaneous configuration along the MD trajectory ( $T=425 \mathrm{~K}$ ) with respect to the mean density. It can be seen that there is no apparent symmetric or directional response in the charge density. c Autocorrelation function of the change in the electrostatic potential energy, which represents a disorder potential for the electronic states and was calculated for the direction indicated in panel b, along the MD simulation at different temperatures. The thin curves show sample snapshots taken along the MD trajectory, and the thick curves their averages. The disorder potential in $\mathrm{CsPbBr}_{3}$ is confined dynamically to very short ranges of nearest-neighbor atomic distances
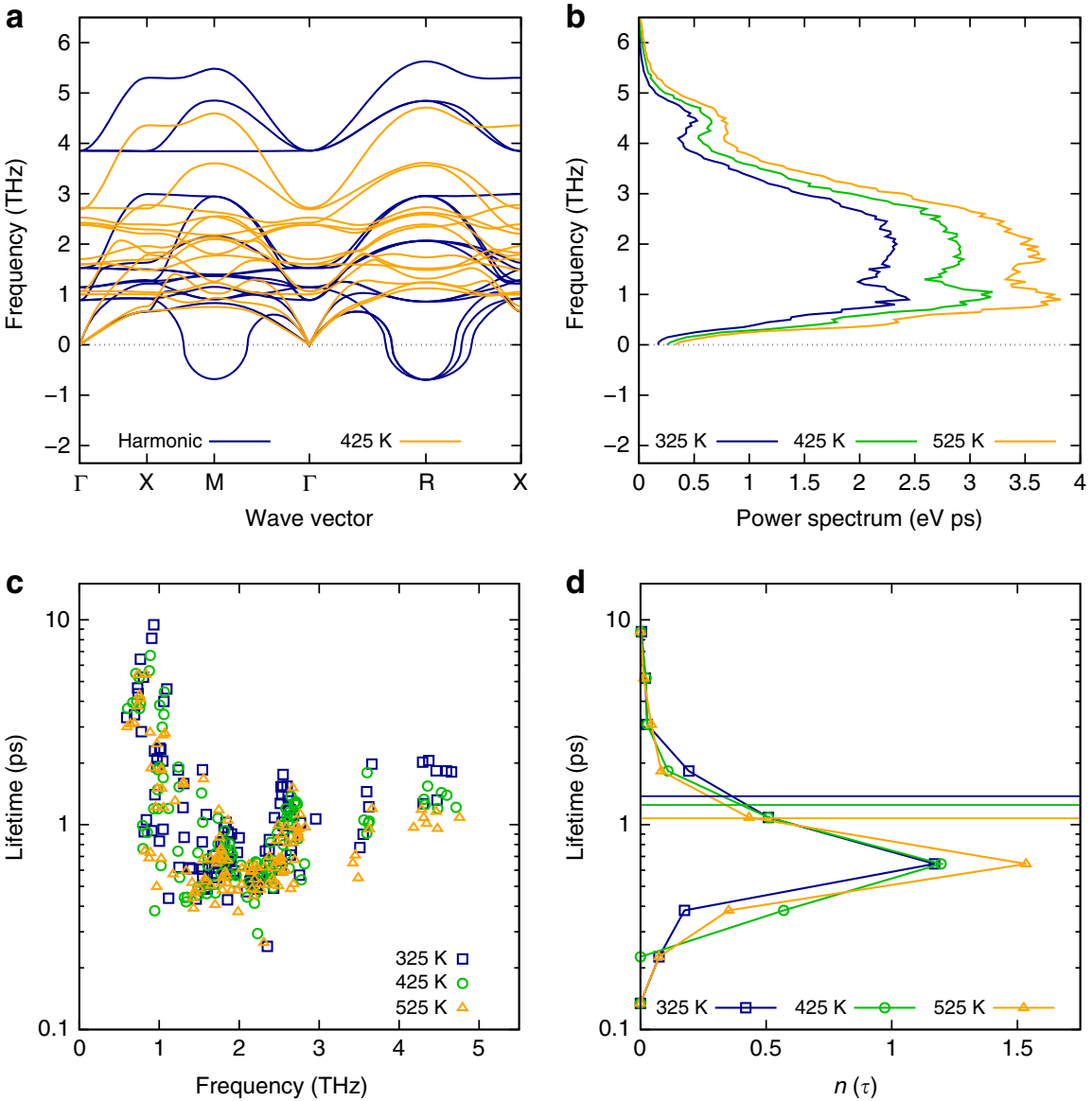

Fig. 2 Vibrational properties and anharmonicity in $\mathrm{CsPbBr}_{3}$. a Harmonic (blue) and finite-temperature renormalized phonon dispersion at $T=425 \mathrm{~K}$ (orange), as obtained from lattice dynamics calculations in the harmonic approximation and fully anharmonic MD, respectively. $\mathbf{b}$ Power spectrum of the velocity-autocorrelation function, as obtained from fully anharmonic MD, calculated at three temperatures. c Phonon lifetime as a function of the renormalized phonon frequency, shown at three temperatures. $\mathbf{d}$ Normalized histogram of the phonon lifetimes, $\tau$, shown for each of the three considered temperatures; see Table 1 for further details. The normalization procedure is such that the integral over the range is 1 . The vertical lines indicate the mean value of the distribution at each temperature, which are $1.38,1.25$, and 1.08 ps at 325,425 , and 525 , respectively

finite temperature vibrational properties of $\mathrm{CsPbBr}_{3}$ obtained from a fully anharmonic treatment by means of DFT-based MD, analyzing the VACF as described in the Methods section. The structural instabilities, seen as imaginary features in the $0 \mathrm{~K}$ harmonic calculations of the phonon spectrum, vanish in the finite temperature dispersion relation (see Fig. 2a), which is expected. Figure $2 \mathrm{~b}$ shows the power spectrum of the VACF, which represents the vibrational density of states (VDOS), at three temperatures. Overall, while the vibrational features appear strongly broadened in the finite temperature spectra, one can still 
appreciate that they are rather similar to the harmonic result. Hence, theoretical data obtained from lattice dynamics calculations in the harmonic approximation are not generally in contrast to those obtained with a fully anharmonic treatment.

This motivates us to study the consequences of anharmonicity in greater detail, especially because the importance of it for optoelectronic properties was discussed in several previous articles $^{13-25}$. Our approach is intrinsically based on firstprinciples information that is obtained at each timestep of the MD trajectory. Therefore, we are in a position to compute phonon quasiparticle properties and extract the vibrational lifetimes, which are key physical observables quantifying anharmonic nuclear effects. The phonon lifetimes, shown in Fig. $2 \mathrm{c}$ at different high symmetry points of the phonon branches as a function of frequency, are extremely short in $\mathrm{Cs} \mathrm{PbBr}_{3}$, i.e., between 0.3 and $10 \mathrm{ps}$ in a temperature range of $325-525 \mathrm{~K}$. We also find that the phonon lifetime is shortest in the frequency range where the power spectrum exhibits the highest intensity, i.e., between 1 and $3 \mathrm{THz}$, which can be explained by considering that due to the presence of many phonons in this range the phonon-phonon interactions are strongest (cf. Fig. 2a-c). Table 1 establishes that both acoustic and optical phonons are very shortlived in $\mathrm{CsPbBr}_{3}$, in agreement with recent experiments that studied the phonon lifetimes of acoustic modes in $\mathrm{MAPbI}_{3}{ }^{25}$.

Figure $2 \mathrm{~d}$ shows histograms of the phonon lifetimes as a function of temperature. Some temperature-induced changes are indeed observed in the phonon lifetimes, e.g., certain modes do shift to lower lifetimes at higher temperatures, and the mean value of the distribution is lowered by approximately 0.3 ps from 325 to $525 \mathrm{~K}$. Notably, however, the shape of the distribution remains largely similar and there is no apparent shift of the distribution to lower lifetimes with increase in temperature. Likewise, Table 1 shows the lower end of the range in the phonon lifetimes to remain largely constant (within a reasonable statistical error of approximately $80 \mathrm{fs}$ ) at different temperatures, while the upper end changes significantly. This finding can be explained by considering that in order to be physically meaningful, the period of a phonon with a given frequency presents a reasonable lower limit to its lifetime. We find that for $\mathrm{CsPBr}_{3}$, this limit has been reached already at $325 \mathrm{~K}$, which again signals massive anharmonic effects in the nuclear displacements approaching the limit of a breakdown of the phonon picture. To put these findings in perspective, recall that in bulk $\mathrm{Si}$ the phonon lifetimes are two orders of magnitude higher for a similar range of temperature and frequency ${ }^{30}$. These findings strongly suggest that anharmonicity is an important phenomenon in the nuclear dynamics of HaPs, in agreement with previous studies on different $\mathrm{HaPs}^{13-25}$. It will now be shown that the mechanism underlying anharmonicity in $\mathrm{HaPs}$ renders the presence of a short-range correlated disorder potential particularly interesting.

Long-range resonant bonding effect in the disorder potential. Resonant bonding was recently studied as a potential origin of the nuclear anharmonicity occurring in $\mathrm{HaPs}^{14}$. Such a mechanism was suggested 40 years ago for hybrid $\mathrm{HaPs}^{31}$ and originates from the fact that multiple configurations of the $s p$-hybridized lead-

Table 1 Range for the lifetimes of acoustic and optical phonons in $\mathrm{CsPbBr}_{3}$, calculated for different temperatures

\begin{tabular}{|lll} 
Temperature (K) & Acoustic modes (ps) & Optical modes (ps) \\
\hline 325 & $0.5-9.5$ & $0.3-4.6$ \\
425 & $0.4-6.7$ & $0.3-4.4$ \\
525 & $0.5-5.4$ & $0.3-2.8$ \\
\hline
\end{tabular}

halide framework are close in energy, as was discussed in previous work on $\mathrm{HaPs}^{14,17}$ and other materials ${ }^{32,33}$. Importantly, resonant bonding causes long-range effects in the dynamical matrix ${ }^{32}$, which we illustrate with an example that was recently studied in the literature ${ }^{14}$. In Fig. 3a we plot the change in the charge density that is induced to the system upon displacing a single $\mathrm{Pb}$ atom showing that the response is clearly long-ranged in $\mathrm{Cs} \mathrm{PbBr}_{3}$. Furthermore, in Fig. $3 \mathrm{~b}$ we show that such a resonant bonding response would result in a long-range correlated disorder potential for electrons and holes in $\mathrm{CsPbBr}_{3}$ by computing $C(\Delta y)$ for this specific case: the long-range effect in the density immediately translates into long-range correlations of the disorder potential that are induced upon the displacement, which exceed the $5.8 \AA$ unit cell of $\mathrm{CsPbBr}_{3}$ by far. In contrast, the full MD calculations discussed above have established a short-range correlated disorder potential that was confined to distances of one atomic bond.

Resolving the discrepancy between long-range disorder effects implied by a resonant bonding mechanism and the appearance of short-range correlated disorder in the actual MD calculations is important. While the role of disorder for properties of solar materials has been long known ${ }^{34}$, revealing the origin and consequences of the short-range correlated disorder present in $\mathrm{HaPs}$ will provide a fresh view on the interrelation of nuclear dynamics and optoelectronic properties in these materials. Consider the case of long-range correlated disorder (see Fig. 4a), defined as being correlated across several atomic bond distances, which would perturb the electronic states in large fractions of the crystal volume. A macroscopic sample of such a material would therefore contain a high density of tail states if long-range correlated disorder was indeed active. The Urbach energy would then be large, since it scales with the square of the correlation length of the disorder potential ${ }^{27}$. In contrast, the type of shortrange correlated disorder we found in $\mathrm{CsPbBr}_{3}$, which is correlated only across distances on the order of one atomic bond, perturbs the electronic states in only small fractions of the crystal volume (see Fig. 4b). Hence, in a macroscopic sample of $\mathrm{CsPbBr}$, different regions of the crystal are perturbed electronically only independently of one another by the nuclear dynamics. Importantly, while locally and temporally the nuclear configuration may generally be strongly perturbed from the ideal lattice, this effect is expected to induce tail states in only a relatively small crystal volume. Therefore, a large crystal sample of $\mathrm{CsPBr}_{3}$ is expected to on average containing only a small density of disorder-induced tail states, the distribution of available band gaps will be narrow, and the optical absorption edge will be sharp. This is a prerequisite for the small Urbach energy that was recorded experimentally for this ${ }^{35}$ and other $\mathrm{HaPs}^{12}$, which is a highly desired property of any solar material. It also implies that the impact of thermal nuclear disorder on the optoelectronic properties of Cs-based $\mathrm{HaPs}$ is such that the optical absorption onset is still sharp. Hence, an efficient collection of sunlight in thin-film devices should be possible given the intrinsic properties of these materials. The mechanism underlying the short-range disorder potential, however, is expected to be different in HaPs compared to classical inorganic semiconductors such as bulk $\mathrm{Si}$ and GaAs. For Si, it was shown that even for a statically displaced structure the response is shortranged $^{14}$, i.e., in $\mathrm{Si}$ it does not require any nuclear dynamics to find a short-range correlated disorder potential.

Dynamic shortening of spatial correlations. We now explore the impact of nuclear dynamics on the spatial correlation in the disorder potential of electrons and holes in more detail. To this end, we consider several relevant constrained scenarios of nuclear 
a

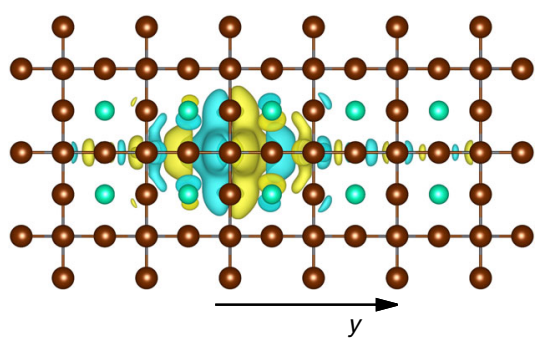

b

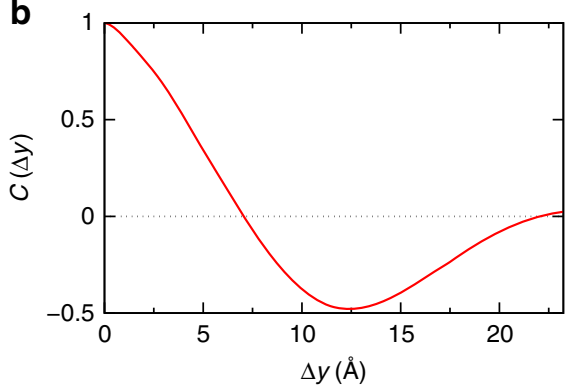

Fig. 3 Resonant bonding in $\mathrm{CsPbBr}_{3}$. a Iso-surface representation of the charge-density difference that is induced in the crystal upon displacing a $\mathrm{Pb}$ atom, which shows a long-range behavior. $\mathbf{b}$ Autocorrelation function of the change in the electrostatic potential energy calculated for the direction indicated in panel a. It represents a disorder potential for the electronic states and also shows a long-range behavior
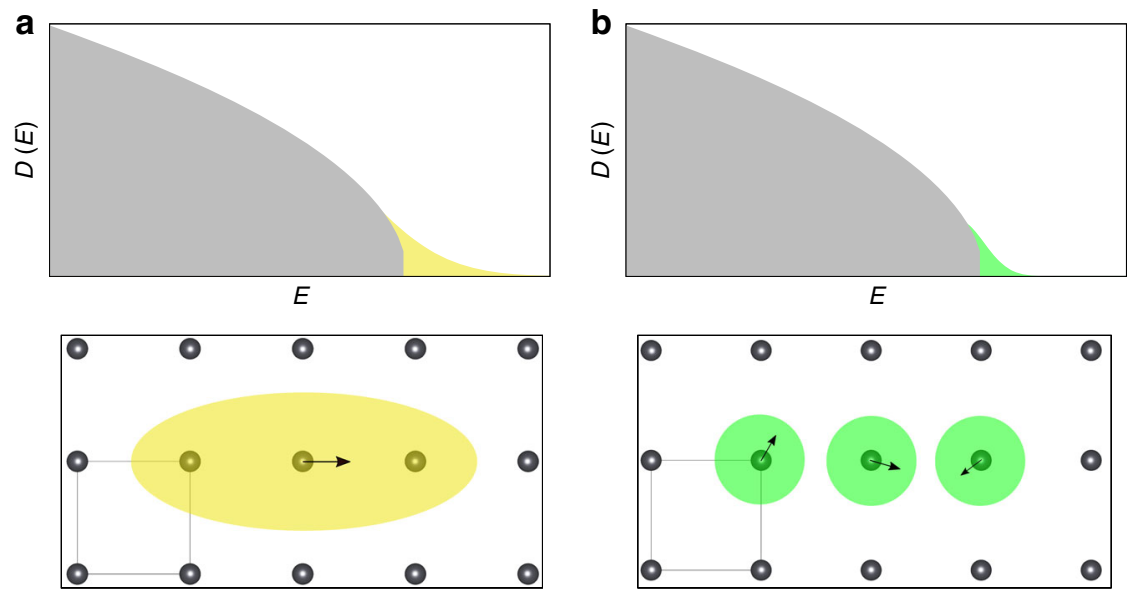

Fig. 4 Impact of differently correlated disorder potentials on the electronic states. a Long-range correlated disorder induced by a single displacement (signified by the arrow) that leads to a long-range disorder potential, illustrated as a yellow disorder domain. It implies a larger amount of tail states in the electronic density of states, $D(E)$, since larger parts of the crystal volume are perturbed by each disordered nuclear configuration. $\mathbf{b}$ Short-range correlated disorder is illustrated by green disorder domains in the lower panels that are characterized by uncorrelated displacements (signified by the arrows). It leads to a small amount of tail states in $D(E)$, since only small parts of the crystal volume are perturbed by each disordered nuclear configuration

motion that occur in the full $\mathrm{MD}$ of $\mathrm{CsPbBr}_{3}$ at $T=425 \mathrm{~K}$. For each considered case, we calculate how it impacts the spatial correlations in the disorder potential of the material. Figure 5a shows data for $C(\Delta y)$ corresponding to three cases: (i) configurations of $\mathrm{Pb}$ atoms chosen from the MD trajectory, but $\mathrm{Cs}$ and $\mathrm{Br}$ atoms being fixed to their ideal lattice positions; (ii) configurations of $\mathrm{Pb}$ and $\mathrm{Br}$ atoms chosen from the $\mathrm{MD}$ trajectory, but only Cs atoms being fixed to their ideal lattice positions; and (iii) configurations of $\mathrm{Pb}$ and $\mathrm{Cs}$ atoms chosen from the $\mathrm{MD}$ trajectory, but only $\mathrm{Br}$ atoms being fixed to their ideal lattice positions. It is found that $\mathrm{Pb}$ displacements induce a disorder potential for the electronic states showing a spatial correlation that significantly exceeds atomic distances, in line with the expectation borne from the resonant bonding mechanism discussed above. Most importantly, the correlation length of the disorder potential for electrons and holes is massively shortened by activating either $\mathrm{Cs}$ or Br displacements. Cs displacements already cause a substantial reduction of the correlation length, but it is the presence of displaced $\mathrm{Br}$ atoms that reduces spatial correlations in the disorder potential such that it becomes similar to the result obtained in fully-unconstrained MD (see Fig. 1c). Hence, the disorder potential for electrons and holes is dynamically shortened by the large nuclear motions of $\mathrm{Cs}$ and especially $\mathrm{Br}$ at elevated temperatures in $\mathrm{CsPbBr}_{3}$.

Narrowing of dynamical band-edge distributions. A manifestation of electron-phonon interactions in $\mathrm{CsPbBr}_{3}$, namely the impact of spatial correlations in the disorder potential on the density of tail states (see Fig. 4), can now be tested explicitly. The investigation of this effect is based on the above finding that when only $\mathrm{Pb}$ displacements are active, the correlation length was found to be longer than in the full MD calculation (cf. Figs. 5a and 1c). In Fig. 5b, we therefore report normalized histograms of DFT-calculated band-edge energies that occur during constrained $\mathrm{MD}$ calculations in which only $\mathrm{Pb}$ displacements were considered, and those of the full MD run (see Methods section for details). First, all finite temperature distributions for the valence band maximum (VBM) and conduction band minimum (CBM) are broadened compared to the static DFT result. This is expected because multiple nuclear configurations are sampled at elevated temperature, which affects the orbital overlap in the perovskite lattice and leads to fluctuations in the instantaneous VBM and $\mathrm{CBM}$ energies. Second, considering the case where only $\mathrm{Pb}$ displacements are active (blue curve in Fig. 5b), it can be seen that the distribution of CBM energies is wider than the one of VBM energies. This finding can be explained by the anti-bonding character of the valence band ${ }^{36}$ and the fact that the long-range nature of resonant bonding is mostly due to $\mathrm{Pb} p$-states, which contribute strongly to the CBM states ${ }^{6}$. Hence, the effect of just $\mathrm{Pb}$ displacements being active in $\mathrm{CsPbBr}_{3}$ leads to tails in the CBM energy distribution, which is again expected. Third and most important, when we now consider the result of the full MD calculation (red-filled curve in Fig. 5b), we find that the distribution of CBM energies becomes much narrower compared to 

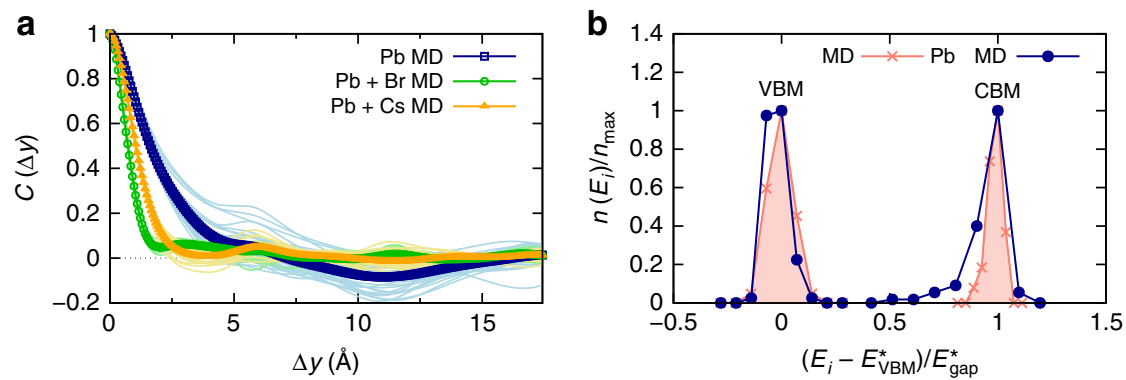

Fig. 5 Shortening of disorder potential and band-edge distributions in $\mathrm{CsPbrr}_{3}$. a Autocorrelation function of the disorder potential corresponding to three scenarios, where the configurations of $\mathrm{Pb}$ atoms were always chosen from the MD simulation $(T=425 \mathrm{~K})$, and: ( $\mathrm{i}$ ) $\mathrm{Cs}$ and $\mathrm{Br}$ atoms were fixed to their ideal lattice positions (blue curve); (ii) configurations of $\mathrm{Br}$ atoms were also chosen from the MD trajectory, but $\mathrm{Cs}$ atoms were fixed to their ideal lattice positions (green curve); and (iii) configurations of $\mathrm{Cs}$ atoms were also chosen from the MD trajectory, but Br atoms were fixed to their ideal lattice positions (orange curve). The thin curves show sample snapshots taken along the MD trajectory $(T=425 \mathrm{~K}$ ), and the thick curves their averages. The longer correlation length in the disorder potential induced by the $\mathrm{Pb}$ displacements is dynamically shortened by the $\mathrm{Cs}$ and especially the $\mathrm{Br}$ displacements. b Normalized histograms of the instantaneous energies, $E_{i}$, of the valence band maximum (VBM) and conduction band minimum (CBM), calculated along two relevant $\mathrm{MD}$ scenarios: constrained configurations for which $\mathrm{Pb}$ atoms were chosen from the $\mathrm{MD}$ simulation $(T=425 \mathrm{~K})$, but all other atoms were fixed to their ideal lattice positions (blue curve with dots); and configurations for which all atoms are displaced according to the MD calculation at $T=$ $425 \mathrm{~K}$ (red curve with stars). $E_{\mathrm{VBM} / \mathrm{CBM}}^{*}$ denotes the VBM/CBM energy with the highest occurrence, $n_{\max }$, which is given by $n_{\max }=n\left(E_{\mathrm{VBM} / \mathrm{CBM}}^{*}\right)$, and $E_{\text {gap }}^{*}=E_{\mathrm{CBM}}^{*}-E_{\mathrm{VBM}}^{*}$; see Methods section for details. The spread in the CBM energy distribution due to the displaced Pb atoms is reduced by the presence of other displacements occurring in the material

the case where only $\mathrm{Pb}$ displacements were active. This finding reveals that the shortening of the disorder potential (cf. Figs. 5a and 1c) manifests itself in a reduction of the density of tails in the CBM energy distribution.

Therefore, we have explicitly established the connection between the short-range correlated disorder potential and favorable distribution of electronic states in $\mathrm{CsPbBr}_{3}$. The finding implies that in absence of defect states and other static perturbations of the crystal, such a short-range correlated disorder potential will result in the recovery of one of the hallmark optoelectronic properties of HaPs, namely a sharp optical absorption edge and small Urbach energy at elevated temperatures. These are key properties of any solar material since they allow for efficient collection of sunlight in thin-film crystalline materials.

Influence of ionic composition. A variety of ionic compositions are compatible with the $A B X_{3}$ stoichiometry of HaP crystals. Several recent experimental and theoretical data showed strong anharmonic and disorder effects for $\mathrm{HaP}$ compounds containing various $A$-site cations and $X$-site anions ${ }^{13-25}$, which nevertheless exhibit favorable optoelectronic properties including a small Urbach energy. Therefore, it is interesting to examine whether the above-described short-range correlated disorder for the electronic states in $\mathrm{CsPbBr}_{3}$ is present also in alternative $\mathrm{HaP}$ compounds. To this end, in a first step we exchange bromine by iodine, and then cesium by the organic methylammonium (MA) molecule, to investigate the disorder potential in the $\mathrm{CsPbI}_{3}$ and $\mathrm{MAPbI}_{3}$ crystal, respectively.

In Fig. 6a, we show $C(\Delta y)$ of $\mathrm{CsPb}_{3}$, calculated for multiple instantaneous nuclear configurations along the MD trajectory $(T$ $=425 \mathrm{~K}$ ) together with their average. It is found that the disorder potential is markedly short-ranged, since it vanishes on the length scale of atomic bonds in $\mathrm{CsPbI}_{3}$, similar to the situation in $\mathrm{CsPbBr}_{3}$ (see Fig. 1c). To investigate the effect of the nuclear dynamics on the disorder in $\mathrm{CsPbI}_{3}$, we perform the same procedure that was described above for $\mathrm{CsPbBr}_{3}$ and consider constrained scenarios of nuclear motion. The results (see Fig. 6b) show that the mechanism of a dynamic shortening of the disorder potential is indeed active also in $\mathrm{CsPbI}_{3}$. In particular, the longerrange disorder induced by $\mathrm{Pb}$ displacements is shortened strongly by activating Cs and especially I displacements. While the mechanism of dynamically shortening the disorder in $\mathrm{CsPbI}_{3}$ is thus remarkably similar to the situation in $\mathrm{CsPbBr}_{3}$ (cf. Figs. 5a and $6 \mathrm{~b}$ ), there is also one interesting difference. The partially negative correlation induced by activating only $\mathrm{Pb}$ displacements in $\mathrm{CsPBr}_{3}$, at a distance of approximately two lattice constants (see Fig. 5a), is hardly visible for the averaged curve in case of $\mathrm{CsPb}_{3}$ (see Fig. 6b). This suggests that a static lattice of iodine atoms provides more screening of the disorder potential than the one of bromines, which is reasonable given the larger atomic polarizability of the former. Similar to the case of $\mathrm{CsPbBr}_{3}$, in Fig. $6 \mathrm{c}$ we demonstrate explicitly that the shortening of the disorder potential corresponds to reducing the spread of CBM energies in $\mathrm{CsPbI}_{3}$. Note that an interesting difference compared to $\mathrm{CsPBr}_{3}$ is that $\mathrm{Pb}$ displacements induce a more symmetric tailing of $\mathrm{CBM}$ energies.

Finally, we consider the hybrid organic-inorganic $\mathrm{HaP}$ $\mathrm{MAPbI}_{3}$, which is interesting because first of all it was recently demonstrated that long-range effects due to a resonant bonding mechanism are active in this material ${ }^{14}$. Furthermore, the anisotropy and dipole moment of the MA molecule could provide means for both, additional long-range order due to oriented dipoles, as well as additional disorder and screening due to disarranged dipoles. In Fig. 7 we show $C(\Delta y)$ of $\mathrm{MAPbI}_{3}$ calculated by combining trajectories obtained in force-field MD with DFT calculations, as described in the Methods section. Our results for $\mathrm{MAPbI}_{3}$ establish that also in this compound the disorder potential due to thermally activated nuclear displacements is short-ranged. This is in line with the established fact that at higher temperatures, the orientation of MA is largely disordered. Note that it is the lacking dipolar order in $\mathrm{MAPbI}_{3}$, which renders the subsequent analysis of constrained $\mathrm{MD}$ scenarios shown above for the Cs-based compounds very challenging. With this, we conclude that the dynamically shortranged disorder potential is present in all considered $\mathrm{HaP}$ compounds, which is relevant for their favorable optoelectronic characteristics.

\section{Discussion}

By means of MD calculations we have reported a theoretical analysis of the disorder potential that is induced by thermal 

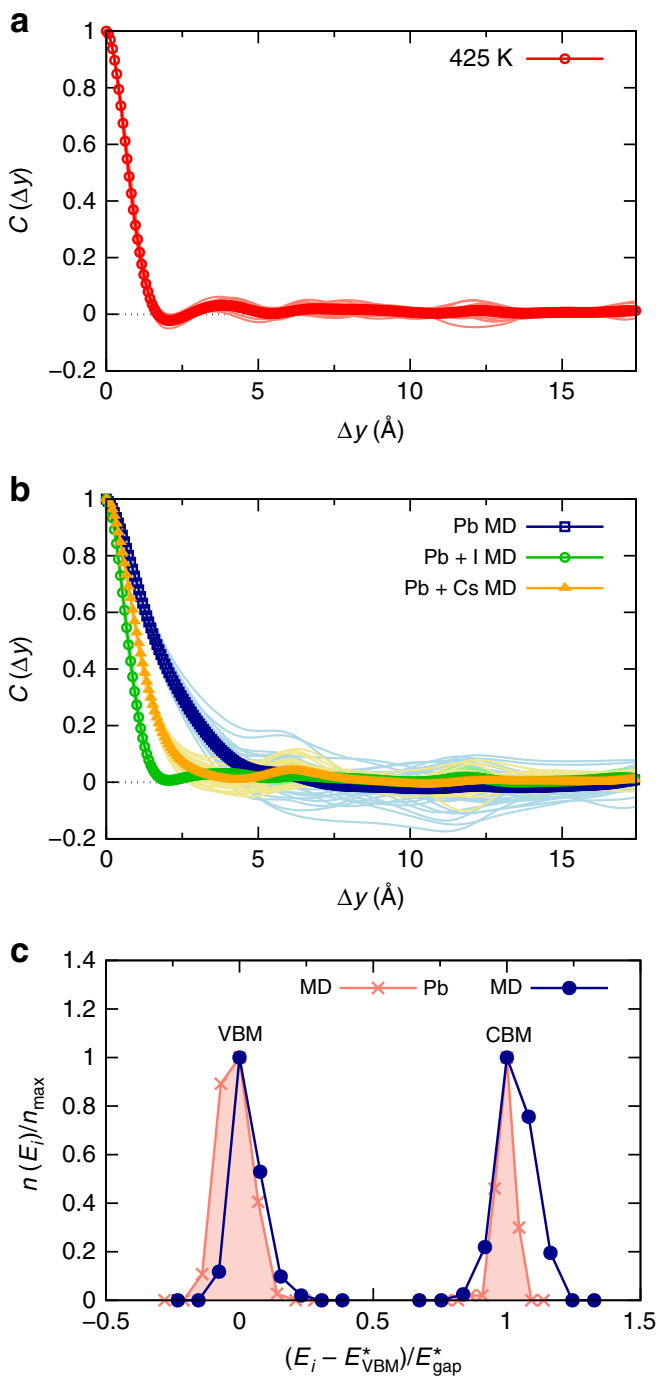

Fig. 6 Shortening of disorder potential and band-edge distributions in $\mathrm{CsPbl}_{3}$. a Autocorrelation function of the disorder potential, where the thin curves show sample snapshots taken along the MD trajectory, and the thick curves shows their average. The disorder potential in $\mathrm{CsPbl}_{3}$ is also confined to very short ranges. $\mathbf{b}$ Autocorrelation function of the disorder potential in $\mathrm{CsPbl}_{3}$ corresponding to the three equivalent scenarios shown in Fig. 5a, where the thin curves show sample snapshots taken along the MD trajectory $(T=425 \mathrm{~K})$, and the thick curves their averages. Also in $\mathrm{CsPb}_{3}$ the correlation length in the disorder potential is dynamically shortened by the Cs and especially the I displacements. c Normalized histograms of the instantaneous VBM and CBM energies calculated equivalently to the data shown Fig. $5 \mathrm{~b}$. In $\mathrm{CsPb}_{3}$ the spread in the CBM energy distribution due to displaced $\mathrm{Pb}$ atoms is also reduced by the presence of the other displacements in the material

vibrations in $\mathrm{HaPs}$ in order to characterize spatial correlation properties. The latter are key for understanding the microscopic origin of the small Urbach energies that were recorded experimentally for $\mathrm{HaP}$ compounds, which enable these materials to be used as efficient solar absorbers in thin-film devices. We found that the correlation length of the disorder potential for electrons and holes is markedly short-ranged in the considered compounds $\mathrm{CsPbBr}{ }_{3}, \mathrm{CsPbI}_{3}$ and $\mathrm{MAPbI}_{3}$, since it was found to be confined to distances of only one atomic bond, which is similar to the lengthscales that were reported for bulk $\mathrm{Si}$ and $\mathrm{GaAs}^{27}$. Notably, this is in agreement with previous findings, which studied the nuclear motion contributing to the central peak in the $\mathrm{CsPbr}_{3}$ Raman

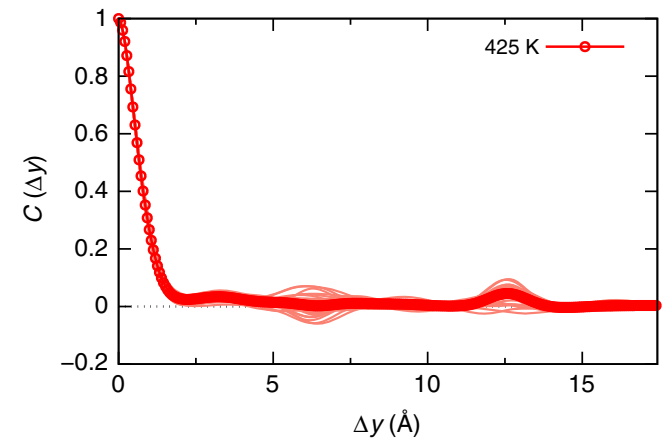

Fig. 7 Characterization of the disorder potential for electronic states in $\mathrm{MAPbl}_{3}$. Autocorrelation function of the disorder potential, where the thin curves show sample snapshots taken along the force-field MD trajectory, and the thick curves shows their average. The disorder potential in $\mathrm{MAPbl}_{3}$ is also confined to very short ranges

spectrum $^{16}$ and quantified the correlation length of the disorder potential in $\mathrm{MAPbI}_{3}$ using classical $\mathrm{MD}^{37}$. The presence of such a short-range correlated disorder potential in $\mathrm{HaPs}$ is peculiar since strongly anharmonic nuclear motions and disorder effects were reported for these systems at room temperature ${ }^{13-25}$, which may be suspected to perturb the electronic disorder in a profound way.

In search for the origin of the short-range correlated disorder potential in HaPs, we considered the microscopic modulations of the electrostatic potential in the material that are induced by the nuclear displacements. Our data showed that a resonant bonding mechanism, which was suggested to be active in HaPs already 40 years ago $^{31}$, is present in these compounds. This result is in strong contrast to the case of classical inorganic semiconductors such as bulk Si or GaAs, but similar to previous findings related to $\mathrm{HaPs}^{14,17}$ and thermoelectric compounds ${ }^{32,33}$. Resonant bonding in HaPs stems from the fact that multiple nuclear configurations of the $s p$-hybridized lead-halide framework are energetically very close, such that nuclear displacements cause long-range effects in the charge density of the system. Since we have also demonstrated that a resonant bonding mechanism would establish long-range correlated disorder potentials for electrons and holes, one must ask how it is possible that HaPs dynamically exhibit a disorder potential that is short-ranged.

To address this question, we could show that it is the massive nuclear motion of the A-site cation and especially the halides leading to a dynamic shortening of the disorder potential induced for electrons and holes. The results from our first-principles calculations explicitly established that the dynamically shortranged disorder potential leads to favorable dynamic distributions of band edge energies. We conclude that it is this dynamic effect, which leads to large crystalline samples of HaPs containing only few disorder-induced tail states. This implies sharp optical absorption edges and low Urbach energies, which are key physical properties of any solar absorber material since they allow for efficient collection of sunlight in thin-film devices.

\section{Methods}

Density functional theory calculations. Density functional theory (DFT) calculations were performed with the plane-wave code $\mathrm{VASP}^{38}$, using the projector augmented wave (PAW) method to treat core-valence interactions ${ }^{39}$. Unless stated otherwise, we employed the "normal" version of the code-supplied PAW potentials. Exchange-correlation was described with the PBE functional ${ }^{40}$, augmented by dispersive corrections computed in the Tkatchenko-Scheffler scheme ${ }^{41}$, which was shown to provide an accurate description of static and dynamic structural properties of $\mathrm{HaPs}^{42}$. Unless stated otherwise, an energy threshold of $10^{-8} \mathrm{eV}$, a $\Gamma$ centered $k$-point grid of $6 \times 6 \times 6$, and a plane-wave cutoff energy set to $500 \mathrm{eV}$ were used. The cubic lattice structure of all compounds were optimized with these settings such that residual forces were below $10^{-3} \mathrm{eV} \AA^{-1}$, for which improved convergence thresholds did not result in any significant changes. This structure was 
used in our subsequent calculations. Structural representations of $\mathrm{Cs} \mathrm{PbBr}_{3}$ were visualized using the VESTA program ${ }^{43}$.

Lattice dynamics. To obtain the phonon dispersion relation and vibrational density of states, lattice dynamics calculations were performed using the finite displacement method as implemented in the phonopy package ${ }^{44}$. In these calculations, a $2 \times 2 \times 2$ supercell of the optimized $\mathrm{CsPbBr}_{3}$ structure was used, and all numerical parameters were kept as before, applying a $k$-point grid that was reduced according to the enlarged cell size.

DFT-based molecular dynamics. First-principles MD calculations were performed using a canonical (NVT) ensemble with a Nosé-Hoover thermostat, as implemented in the VASP code, employing a timestep of $8 \mathrm{fs}$. We have chosen a larger $4 \times 4 \times 2(160$ atom) supercell in the MD simulations in order to improve the statistical sampling of the nuclear dynamics. For making these simulations computationally tractable, more efficient numerical settings were employed: the "GW" PAW potentials were used, since this improved the numerical convergence of the self-consistent calculations of the MD runs, together with a plane-wave cutoff energy of $250 \mathrm{eV}$, an energy threshold of $10^{-6} \mathrm{eV}$, and a single $k$ point. We verified that the latter was sufficiently accurate, by monitoring the power spectrum of the velocity-autocorrelation function calculated with more $k$ points. The system was equilibrated for at least $5 \mathrm{ps}$ at each temperature, and the subsequent nuclear dynamics were analyzed along trajectories of $150 \mathrm{ps}$, which is more than ten times longer than the longest calculated phonon lifetime.

Force-field molecular dynamics. Force-field MD calculations were performed for a $4 \times 4 \times 2$ supercell of cubic $\mathrm{MAPbI}_{3}$ (384 atoms) with the LAMMPS code ${ }^{45}$, applying the force field by Mattoni et al. ${ }^{46}$. We used a canonical (NVT) ensemble with a Nosé-Hoover thermostat, employing a timestep of $0.5 \mathrm{fs}$. The system was equilibrated for $1 \mathrm{~ns}$ followed by a production run of $150 \mathrm{ps}$.

\section{Phonon-quasiparticle properties. Phonon-quasiparticle properties of $\mathrm{Cs} \mathrm{PbBr}_{3}$} were calculated using the dynaphopy package ${ }^{47}$. Specifically, the MD-calculated velocity-autocorrelation functions were analyzed by means of a projection of them onto the harmonic modes which were calculated as described above. Fitting the power spectrum of these mode-resolved projections with Lorentzian functions provided well-defined frequencies and lifetimes of each phonon mode as a function of the phonon wavevector, $\mathbf{q}$, at high symmetry points. Note that the acoustic phonons at the $\Gamma$-point were not included in this analysis, since these are zerofrequency modes. The finite-temperature phonon dispersion was then obtained by calculating renormalized force constants that correspond to the quasiparticle phonon frequencies at the high symmetry points, which provided an updated dynamical matrix that was then interpolated in $\mathbf{q}$, akin to lattice dynamic calculations. Note that the time-frequency transform was achieved by means of standard Fast-Fourier-transform. Further theoretical details can be found in refs. ${ }^{47,48}$.

Disorder potential and band-edge histogram calculations. To calculate the disorder potential and band-edge histograms, we selected instantaneous nuclear configurations along the MD trajectories and computed the electrostatic potential energy and electronic structure of the system self-consistently with DFT. To enhance the accuracy of these calculations, compared to the DFT-MD simulations we increased the cutoff energy to $500 \mathrm{eV}$ and used a $k$-point grid of $1 \times 1 \times 2$ in accordance with the real-space dimensions of the supercell. The autocorrelation function, which we denoted as $C(\Delta y)$, was calculated as described above (see Eqs. 1-3). $C(\Delta y)$ shown in Fig. 3b was obtained in an equivalent way, namely by calculating the disorder potential as the deviation of the electrostatic potential energy of the considered nuclear configuration (one $\mathrm{Pb}$ atom displaced by $5 \%$ of the primitive lattice constant) from the potential energy of the ideal one. In the latter calculations, we used a $5 \times 5 \times 2$ supercell to minimize boundary effects that are induced by the long-range nature of the density response. $C(\Delta y)$ shown in Figs. $5 \mathrm{a}, 6 \mathrm{~b}$, and 7 was also obtained equivalently, namely by calculating the disorder potential as the deviation of the electrostatic potential energy of the considered nuclear configuration (see Figs. 5a, 6b, and 7) from the potential energy of the one averaged for all considered configurations. To compute the band-edge distributions, 90 structures separated by 200 steps or $1.6 \mathrm{ps}$ in the MD were considered. The normalization of the histograms was performed for the valence band maximum and conduction band minimum of each type of MD calculation separately. To sacrifice detail for clarity, we have shown the histograms as a function of a dimensionless parameter.

\section{Data availability}

The data that support the findings of this study are available from the corresponding author upon reasonable request.

Received: 10 February 2019 Accepted: 17 June 2019

Published online: 17 July 2019

\section{References}

1. Kojima, A., Teshima, K., Shirai, Y. \& Miyasaka, T. Organometal halide perovskites as visible-light sensitizers for photovoltaic cells. J. Am. Chem. Soc. 131, 6050-6051 (2009).

2. Lee, M. M., Teuscher, J., Miyasaka, T., Murakami, T. N. \& Snaith, H. J. Efficient hybrid solar cells based on meso-superstructured organometal halide perovskites. Science 338, 643-647 (2012).

3. Burschka, J. et al. Sequential deposition as a route to high-performance perovskite-sensitized solar cells. Nature 499, 316-319 (2013).

4. Liu, M., Johnston, M. B. \& Snaith, H. J. Efficient planar heterojunction perovskite solar cells by vapour deposition. Nature 501, 395-398 (2013).

5. Stranks, S. D. \& Snaith, H. J. Metal-halide perovskites for photovoltaic and light-emitting devices. Nat. Nanotechnol. 10, 391 (2015).

6. Brenner, T. M., Egger, D. A., Kronik, L., Hodes, G. \& Cahen, D. Hybrid organic-inorganic perovskites: low-cost semiconductors with intriguing charge-transport properties. Nat. Rev. Mater. 1, 15007 (2016).

7. Sutherland, B. R. \& Sargent, E. H. Perovskite photonic sources. Nat. Photonics 10, 295-302 (2016)

8. Correa-Baena, J.-P. et al. Promises and challenges of perovskite solar cells. Science 358, 739-744 (2017).

9. Li, W. et al. Chemically diverse and multifunctional hybrid organic-inorganic perovskites. Nat. Rev. Mater. 2, 16099 (2017).

10. Tsai, H. et al. Light-induced lattice expansion leads to high-efficiency perovskite solar cells. Science 360, 67-70 (2018).

11. Stoumpos, C. C., Malliakas, C. D. \& Kanatzidis, M. G. Semiconducting tin and lead iodide perovskites with organic cations: phase transitions, high mobilities, and near-infrared photoluminescent properties. Inorg. Chem. 52, 9019-9038 (2013).

12. De Wolf, S. et al. Organometallic halide perovskites: sharp optical absorption edge and its relation to photovoltaic performance. J. Phys. Chem. Lett. 5, 1035-1039 (2014).

13. Whalley, L. D., Skelton, J. M., Frost, J. M. \& Walsh, A. Phonon anharmonicity, lifetimes, and thermal transport in $\mathrm{CH}_{3} \mathrm{NH}_{3} \mathrm{PbI}_{3}$ from many-body perturbation theory. Phys. Rev. B 94, 220301 (2016).

14. Zhu, T. \& Ertekin, E. Mixed phononic and non-phononic transport in hybrid lead halide perovskites: glass-crystal duality, dynamical disorder, and anharmonicity. Energy Environ. Sci. 12, 216-229 (2019).

15. Beecher, A. N. et al. Direct observation of dynamic symmetry breaking above room temperature in methylammonium lead iodide perovskite. ACS Energy Lett. 1, 880-887 (2016).

16. Yaffe, O. et al. Local polar fluctuations in lead halide perovskite crystals. Phys. Rev. Lett. 118, 136001 (2017).

17. Laurita, G., Fabini, D. H., Stoumpos, C. C., Kanatzidis, M. G. \& Seshadri, R. Chemical tuning of dynamic cation off-centering in the cubic phases of hybrid tin and lead halide perovskites. Chem. Sci. 8, 5628-5635 (2017).

18. Brown, K. L. et al. Molecular orientational melting within a lead-halide octahedron framework: the order-disorder transition in $\mathrm{CH}_{3} \mathrm{NH}_{3} \mathrm{PbBr}_{3}$. Phys. Rev. B 96, 174111 (2017).

19. Carignano, M. A., Aravindh, S. A., Roqan, I. S., Even, J. \& Katan, C. Critical fluctuations and anharmonicity in lead iodide perovskites from molecular dynamics supercell simulations. J. Phys. Chem. C. 121, 20729-20738 (2017).

20. Marronnier, A. et al. Structural instabilities related to highly anharmonic phonons in halide perovskites. J. Phys. Chem. Lett. 8, 2659-2665 (2017).

21. Marronnier, A. et al. Anharmonicity and disorder in the black phases of cesium lead iodide used for stable inorganic perovskite solar cells. ACS Nano 12, 3477 (2018).

22. Rivett, J. P. H. et al. Long-lived polarization memory in the electronic states of lead-halide perovskites from local structural dynamics. Nat. Commun. 9, 3531 (2018).

23. Wang, C., Liu, Y., Liu, S. F., Li, B. \& Chen, Y. Giant phonon tuning effect via pressure-manipulated polar rotation in perovskite $\mathrm{MAPbI}_{3}$. J. Phys. Chem. Lett. 9, 3029-3034 (2018).

24. Wang, M. \& Lin, S. Anisotropic and ultralow phonon thermal transport in organic-inorganic hybrid perovskites: atomistic insights into solar cell thermal management and thermoelectric energy conversion efficiency. Adv. Funct. Mater. 26, 5297-5306 (2016).

25. Gold-Parker, A. et al. Acoustic phonon lifetimes limit thermal transport in methylammonium lead iodide. Proc. Natl Acad. Sci. USA 115, 11905-11910 (2018).

26. Egger, D. A. et al. What remains unexplained about the properties of halide perovskites? Adv. Mater. 30, 1800691 (2018).

27. Greeff, C. W. \& Glyde, H. R. Anomalous urbach tail in GaAs. Phys. Rev. B 51, 1778-1783 (1995).

28. Yang, R. X., Skelton, J. M., da Silva, E. L., Frost, J. M. \& Walsh, A. Spontaneous octahedral tilting in the cubic inorganic cesium halide perovskites $\mathrm{CsSnX}_{3}$ and $\mathrm{CsPbX}_{3}(\mathrm{X}=\mathrm{F}, \mathrm{Cl}, \mathrm{Br}, \mathrm{I})$. J. Phys. Chem. Lett. 8, 4720-4726 (2017).

29. Guo, P. et al. Polar fluctuations in metal halide perovskites uncovered by acoustic phonon anomalies. ACS Energy Lett. 2, 2463-2469 (2017). 
30. Henry, A. S. \& Chen, G. Spectral phonon transport properties of silicon based on molecular dynamics simulations and lattice dynamics. J. Comput. Theor. Nanosci. 5, 141-152 (2008).

31. Weber, D. $\mathrm{CH}_{3} \mathrm{NH}_{3} \mathrm{PbX}_{3}$, a $\mathrm{Pb}(\mathrm{II})$-system with cubic perovskite structure. $Z$. Naturforsch. B 33, 1443-1445 (1978).

32. Lee, S. et al. Resonant bonding leads to low lattice thermal conductivity. Nat. Commun. 5, 3525 (2014)

33. Yue, S.-Y., Xu, T. \& Liao, B. Ultralow thermal conductivity in a twodimensional material due to surface enhanced resonant bonding. Mater. Today Phys. 7, 89-95 (2018).

34. Nayak, P. K., Garcia-Belmonte, G., Kahn, A., Bisquert, J. \& Cahen, D. Photovoltaic efficiency limits and material disorder. Energy Environ. Sci. 5, 6022 (2012).

35. Rakita, Y. et al. Low-temperature solution-grown $\mathrm{CsPbBr}_{3}$ single crystals and their characterization. Cryst. Growth Des. 16, 5717-5725 (2016).

36. Yin, W.-J., Shi, T. \& Yan, Y. Unusual defect physics in $\mathrm{CH}_{3} \mathrm{NH}_{3} \mathrm{PbI}_{3}$ perovskite solar cell absorber. Appl. Phys. Lett. 104, 063903 (2014).

37. Mayers, M. Z., Tan, L. Z., Egger, D. A., Rappe, A. M. \& Reichman, D. R. How lattice and charge fluctuations control carrier dynamics in halide perovskites. Nano Lett. 18, 8041-8046 (2018).

38. Kresse, G. \& Furthmüller, J. Efficient iterative schemes for ab initio totalenergy calculations using a plane-wave basis set. Phys. Rev. B 54, 11169-11186 (1996).

39. Kresse, G. \& Joubert, D. From ultrasoft pseudopotentials to the projector augmented-wave method. Phys. Rev. B 59, 1758-1775 (1999).

40. Perdew, J. P., Burke, K. \& Ernzerhof, M. Generalized gradient approximation made simple. Phys. Rev. Lett. 77, 3865-3868 (1996).

41. Tkatchenko, A. \& Scheffler, M. Accurate molecular van der waals interactions from ground-state electron density and free-atom reference data. Phys. Rev. Lett. 102, 073005 (2009).

42. Beck, H., Gehrmann, C. \& Egger, D. A. Structure and binding in halide perovskites: analysis of static and dynamic effects from dispersion-corrected density functional theory. APL Materials 7, 021108 (2019).

43. Momma, K. \& Izumi, F. VESTA 3 for three-dimensional visualization of crystal, volumetric and morphology data. J. Appl. Crystallogr. 44, 1272-1276 (2011).

44. Togo, A., Chaput, L. \& Tanaka, I. Distributions of phonon lifetimes in Brillouin zones. Phys. Rev. B 91, 094306 (2015).

45. Plimpton, S. Fast parallel algorithms for short-range molecular dynamics. $J$. Comput. Phys. 117, 1-19 (1995).

46. Mattoni, A., Filippetti, A., Saba, M. I. \& Delugas, P. Methylammonium rotational dynamics in lead halide perovskite by classical molecular dynamics: the role of temperature. J. Phys. Chem. C. 119, 17421-17428 (2015).

47. Carreras, A., Togo, A. \& Tanaka, I. DynaPhoPy: a code for extracting phonon quasiparticles from molecular dynamics simulations. Comput. Phys. Commun. 221, 221-234 (2017)
48. Zhang, D.-B., Sun, T. \& Wentzcovitch, R. M. Phonon quasiparticles and anharmonic free energy in complex systems. Phys. Rev. Lett. 112, 058501 (2014).

\section{Acknowledgements}

We thank Omer Yaffe and David Cahen (both Weizmann Institute of Science) for fruitful discussions. Funding provided by the Alexander von Humboldt-Foundation in the framework of the Sofja Kovalevskaja Award, endowed by the German Federal Ministry of Education and Research, is acknowledged. The authors gratefully acknowledge the Gauss Centre for Supercomputing e.V. for funding this project by providing computing time through the John von Neumann Institute for Computing on the GCS Supercomputer JUWELS at Jülich Supercomputing Centre.

\section{Author contributions}

C.G. performed the theoretical calculations and analyzed the data. D.A.E. conceived and supervised the project. C.G. and D.A.E. interpreted the results and wrote the paper.

\section{Additional information}

Competing interests: The authors declare no competing interests.

Reprints and permission information is available online at http://npg.nature.com/ reprintsandpermissions/

Peer review information: Nature Communications thanks Paulina Plochocka and other anonymous reviewer(s) for their contribution to the peer review of this work.

Publisher's note: Springer Nature remains neutral with regard to jurisdictional claims in published maps and institutional affiliations.

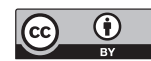

Open Access This article is licensed under a Creative Commons Attribution 4.0 International License, which permits use, sharing, adaptation, distribution and reproduction in any medium or format, as long as you give appropriate credit to the original author(s) and the source, provide a link to the Creative Commons license, and indicate if changes were made. The images or other third party material in this article are included in the article's Creative Commons license, unless indicated otherwise in a credit line to the material. If material is not included in the article's Creative Commons license and your intended use is not permitted by statutory regulation or exceeds the permitted use, you will need to obtain permission directly from the copyright holder. To view a copy of this license, visit http://creativecommons.org/ licenses/by/4.0/.

(C) The Author(s) 2019 